\title{
Saberes y prácticas en la agonía, muerte y duelo del pueblo afrodescendiente de Puerto Cabezas
}

\author{
Knowings and practices in the agony, death and mourning of \\ Puerto Cabezas Afro-descendant people
}

\author{
Dixie Dean Lee Smith ${ }^{1}$ \\ Sandra Carolina Rojas Hooker ${ }^{2}$
}

\section{Resumen}

Este artículo se basó en el estudio: Historia oral sobre las tradiciones culturales: agonía, muerte y duelo en el pueblo afrodescendiente de Puerto Cabezas, con énfasis en una serie de ritos, asociados a etapas o hitos, que el pueblo afrodescendiente realiza de manera particular como un mecanismo colectivo de espiritualidad, acompañamiento, solidaridad y comunitariedad, para el manejo del duelo. Además de compartir sus saberes y práctica; también expresaron sus profundos sentimientos alrededor de algunas prácticas culturales en las nuevas generaciones, así como la posibilidad de revitalizarlas.

Palabras clave: Tradiciones; cultura; agonía; muerte y duelo; afrodescendientes.

\section{Abstract}

This article was based on the study: Oral history of cultural traditions: agony, death and mourning in the Afro-descendant people of Puerto Cabezas, with emphasis on a series of rites, associated with stages or milestones, that the Afro-descendant people perform in a particular way as a collective mechanism of spirituality, accompaniment, solidarity and community, for the mourning management. In addition to sharing their knowings and practices; they also expressed their deep feelings about some cultural practices in the new generations, as well as the possibility of revitalizing them.

Keywords: Traditions; culture; agony; death and mourning: afrodescendants.

\section{Introducción}

El presente trabajo tiene el propósito de compartir algunas historias orales sobre el proceso de agonía, muerte y duelo en el pueblo afrodescendiente de Puerto Cabezas

1 Técnico Superior en Desarrollo Integral. Coordinador del Instituto de Estudios y Promoción de la Autonomía de la Universidad de las Regiones Autónomas de la Costa Caribe Nicaragüense-Recinto Universitario Bilwi. Correo: duhindo@yahoo.com. Orcid: https://orcid.org/oooo-0002-9592-4585

2 Máster en Estudios Indígenas. Directora del Instituto de Estudios y Promoción de la Autonomía de la Universidad de las Regiones Autónomas de la Costa Caribe Nicaragüense. Correo: sandra.rojas@uraccan.edu.ni ORCID: https://orcid.org/oooo-0002-7467-2366

Recibido: 05/02/2018 Aprobado: 30/04/2018 
(en la Región Autónoma de la Costa Caribe Norte de Nicaragua), que nos permitirá conocer, entender y valorar aún más, la riqueza cultural al compartir un rito de pasaje que marcan al individuo, la familia y colectividad. Así como las percepciones religiosas, espirituales y filosóficas alrededor del tema. Estas tradiciones se encuentran en la memoria colectiva y consideramos necesario hacerlo visible en el presente trabajo, para su práctica, conocimiento y perpetuidad.

La muerte, va acompañado de rituales de separación por medio del cual, el individuo pasa a un nuevo estado ya definitivo, incorporándose a un mundo que no es de los vivos y que genera: 1) una serie de actitudes y formas de conducta culturalmente establecidas. 2) Un cambio en los procesos de interacción a nivel familiar y social. 3) una ideología resultante que puede explicarse a través de los hechos descritos a ser vehículos de análisis para el resto de los componentes de la cultura ${ }^{3}$.

A través de la historia oral, se tuvo un registro fiel de la comunicación de las personas, su lenguaje y formas propias de interpretar estas vivencias, lo que permitió reconocer las significaciones que le dan y compartir las experiencias y sentimientos en un escrito con los Pueblos Indígenas, Afrodescendientes, Mestizos y Comunidades Étnicas. Los hallazgos del estudio, es una oportunidad de una nueva mirada y entendimiento al manejo de las diferentes etapas del duelo y los mecanismos de superación desde la cosmovisión Afrodescenciente.

\section{Revisión de la literatura}

El concepto de cultura, es uno de los más difíciles de entender porque tiene múltiples abordajes desde diversas disciplinas científicas que tratan de integrar una definición común. No obstante, más allá de su definición, lo cierto es que no podemos hablar de la existencia de una cultura sino, de una diversidad de culturas que conviven e interactúan en un espacio determinado. La Organización de las Naciones Unidas para la Educación, la Ciencia y la Cultura (UNESCO) en la Conferencia Mundial sobre Políticas Públicas desarrollada en 1982 señala:

La cultura puede considerarse actualmente como el conjunto de los rasgos distintivos, espirituales y materiales, intelectuales y afectivos que caracterizan a una sociedad o un grupo social. Ella engloba, además de las artes y las letras, los modos de vida, los derechos fundamentales al ser humano, los sistemas de valores, las tradiciones y las creencias y que la cultura da al hombre la capacidad de reflexionar sobre sí mismo. Es ella la que hace de nosotros seres específicamente humanos, racionales, críticos y éticamente comprometidos. A través de ella discernimos los valores y efectuamos opciones. A través de ella el hombre se expresa, toma conciencia de sí mismo, se

3 Allue Martínez, Marta (1982) Rituales Funerarios. Institut Catala d’ Antropología, Tarragona. Axiu D’etnografia de Catalunya, No 2. 1982. P.134 
reconoce como un proyecto inacabado, pone en cuestión sus propias realizaciones, busca incansablemente nuevas significaciones, y crea obras que lo trascienden.

La cultura, por tanto, "no es un instrumento del progreso material: es el fin y el objetivo del desarrollo, entendido en el sentido de realización de la existencia humana en todas sus formas y en toda su plenitud" (UNESCO, 1996).

Como parte de la construcción social, las personas se han encargado de darle una explicación a todo aquello que no han podido entender, por medio de los mitos. Narran historias para darle salida a ciertos fenómenos que surgen y presentan en el mundo. En el caso de la muerte, ésta no ha sido la excepción. A lo largo de la historia, cada cultura ha construido sus propias creencias y con ellas las ceremonias que las acompañan, que generalmente, al igual que los mitos de la antigüedad, están relacionadas a la religión.

En la actualidad, la inquietud ante la muerte sigue presente, y con ello, en todo el mundo se encuentran creencias y tradiciones alrededor de ella, relacionadas a las diversas formas que la gente tiene para actuar cuando se presenta. Como construcción social, la muerte nos sitúa ante las diversas formas en que las sociedades elaboran el sentido de vida, su visión, vivencia, y sus prácticas mortuorias asociadas. Representan la manera en que cada cultura ha decidido adaptarse a su entorno y sus posibilidades de trascendencia.

Así, el Pueblo Afrodescendiente ha ido construyendo un conjunto de creencias en torno a la muerte y el rito de transición o pasaje que constituyen actos individuales y colectivos que deben sujetarse fielmente a ciertas reglas y que se caracterizan por su capacidad expresiva, de repetición, simbología y adscripción a una determinada religión en el que participa la colectividad. Esto con el fin de realzar el sentido de solidaridad y acompañamiento de la comunidad como un aspecto primordial para superar las transiciones vitales de carácter social y natural que nos impone el destino o la existencia misma.

Las ceremonias comunitarias de transición o pasaje al más allá, se practican en todas las culturas para tener una explicación de los diferentes momentos de cambio por la que pasan cada miembro de la sociedad en este proceso. La principal función de estos ritos es reconocer los cambios que experimentan las personas en lo individual y en lo social para brindar una estructura, orden y sentido a la existencia de los seres humanos, mediante el desarrollo de ceremonias periódicas, formales y participativas que regulan las relaciones entre las personas y las culturas a partir de la dicotomía vida/muerte que subyace en la práctica de rituales funerarios. 


\section{Materiales y métodos}

La metodología que se utilizó fue cualitativo encaminado al reconocimiento de situaciones y comportamientos mediante la historia oral para identificar valores, creencias, normas y prácticas del pueblo Afrodescendiente, mediante la interacción con los participantes. Se realizó grupos focales y entrevistas en donde participaron diferentes personalidades, sectores sociales y religiosos, jóvenes, adultos y ancianos de ambos sexos. Los resultados fueron leídos, analizados y organizados en base a las etapas e hitos del duelo Afrodescendiente que inician con las señales o anticipos, fallecimiento y velorio en casa, entierro, nueve días y culmina en algunos casos con la ritualidad después de un año de fallecida la persona.

\section{Resultados y discusión}

Todos nacemos dentro de una cultura determinada, cuyas creencias son incorporadas a medida que vamos creciendo. Aprendemos el significado de la muerte, la que se ve condicionado por las creencias culturales transmitidas por la familia, sociedad y pueblo al que pertenecemos. No obstante, hasta que perdemos un ser querido, el sentimiento y el dolor verdadero por la pérdida, se hacen realidad.

El proceso de agonía, muerte y duelo, responden al sistema, donde se presenta, articula y entreteje una serie de actividades que generan sentido para el Pueblo Afrodescendiente. A continuación, presentaremos algunos elementos para comprender el abordaje espiritual, los saberes y haceres, roles y la relación que entrelaza a las partes en el desarrollo de este fenómeno cultural.

\section{Señales 0 anticipos}

Un primer hito es la presencia de señales o anticipos que anuncian la muerte. Los mensajeros más frecuentes de la muerte en la creencia Afrodescendiente son los animales, tal es el caso del canto de un búho, el maullido de los gatos, muerte repentina de un animal casero y el ladrido lastimero de un perro. Hay quienes ven o perciben fantasmas, ya que el espíritu deambula antes de la muerte de la persona; la visita del macking bird (ruiseñor) que entra a la casa y vuela en cuatro direcciones, formando una cruz en señal de que habrá una muerte repentina de un familiar cercano, aun cuando no hay enfermos en la casa. Cuando escuchamos a una gallina imitando el canto de un gallo, significa que la muerte lo ronda, si dos gallinas cacarean con un gallo, entonces quiere decir que morirá una pareja y si el gallo canta en la noche o en la madrugada algo malo o trágico está por ocurrir.

El más usual es la visita de una mariposa de color negro que entra por la puerta principal y sale por la trasera o viceversa, esto representa la muerte de un familiar o un amigo cercano en las próximas horas. Recalcan que esta mariposa no se ve todos 
los días y que se diferencia claramente cuando es símbolo de visita o muerte. También relacionan los anticipos o señales con la visita o apariciones de familiares ya fallecidos que generalmente es identificado como la llamanda, visita del espíritu de los ancestros. Estos vienen a afirmarles que todo estará bien, a brindarles paz y tranquilidad para el proceso de transición de una vida a otra.

El marchitar repentino de un árbol frondoso en el patio, rompimiento de un espejo o vaso de vidrio en la casa sin haberlo tocado, un fuerte viento que ocasiona el cierre brusco de puertas y ventanas seguido de un escalofrío que recorre todo el cuerpo, es aviso de muerte de un familiar o amigo cercano. Algunos entrevistados también refieren que puede haber apariciones de niños o ángeles caminando en la pared de la casa como señal que algo anda mal, aún más en período de Semana Santa.

Se cree también que, en viernes santo, si se abre un huevo de amor en un vaso de vidrio con agua y se coloca en el sol desde las seis de la mañana dejándolo reposar hasta mediodía, esta presenta diferentes figuras que va desde una Iglesia, cementerio, ataúd, barco, casa, y otros. Si la figura formada es la de un ataúd, o un cementerio, es augurio que alguien de la familia muy pronto morirá.

Las señales o anticipos, también pueden darse a través de la interpretación de sueños, siendo los de mayor referencia una casa nueva, cerrada, vacía o un bote o dori, que representa un ataúd, o la caída de un diente. Si es el diente colmillo "eye tooth," eso significa la muerte de familiares en línea recta: padres, hermanos e hijos. La caída de cualquier otro diente puede significar la muerte de un amigo cercano o conocido.

El grupo focal, hizo referencia a otras señales que anuncian la muerte de un familiar que está en su lecho de muerte, tal es el caso de la pérdida de apetito. Una gestora de la cultura creole expresa:

En la cultura creole, tenemos nuestras creencias. Cuando la muerte ronda, lo sentimos en lo más profundo de nuestro ser. Lo sentimos en nuestro cuerpo por días, nos invade una sensación de tristeza y no observamos mejoría alguna de nuestro ser querido, lo sientes, duele, duele muchísimo.

La persona enferma empieza a resistirse o negarse a comer o beber y solo se presta a tomar pequeñas cantidades de alimentos como leche o un sorbo de agua para quitarse la sed. En estos casos los familiares humedecen el contorno de los labios del enfermo con un poco de agua o toalla húmeda o utilizan un poco de vaselina para que no se agrieten y les duela. Presentan un exceso de sueño y fatiga, debilitamiento hasta el punto de volverse incapaces de levantar la cabeza o incluso de comer.

Los familiares procuran que la persona se siente confortable y acompañado en estos momentos. Cuando los órganos del cuerpo empiezan a dejar de funcionar, 
produce confusión en la persona enferma, dejan de saber dónde están con exactitud y quienes están en la habitación. La persona que está agonizando empieza a decir cosas sin sentido, a agitarse, a ver cosas, se le dificulta la respiración y a producir ruidos fuertes al respirar, llamado estertor o rattling, en inglés. Estos son momentos estrictamente familiares y preparatorios del desenlace ya previsto, tanto para el enfermo como para su familia quienes lo rodean. En estos momentos comienzan a cantarle, le hablan suavemente y tratan que la persona enferma se sienta cómoda.

El acompañamiento de los vecinos y la comunidad es fundamental, ya que se percibe la unidad y solidaridad de la red comunitaria. La parte espiritual juega un rol importante, el acompañamiento del líder religioso y los familiares brinda la posibilidad de iniciar el proceso de duelo anticipado, de poder prepararse para la muerte de su ser querido.

Al ser testigo del debilitamiento progresivo de la persona, junto con la creciente impotencia personal para detenerlo, genera muchas veces angustia, dolor y una honda sensación de vacío y pérdida, pero le da a la familia, los amigos y la comunidad, una oportunidad a prepararse para enfrentar los gastos que conlleva a la muerte.

\section{Fallecimiento y velorio en casa}

El velorio es una práctica que tiene lugar unas horas después del fallecimiento de la persona. En la Costa Caribe de Nicaragua es habitual que se lleve a cabo en la casa o en algún otro establecimiento vinculado a la actividad u origen del difunto.

Cuando recién muere el ser querido, comienzan a aparecer los amigos y familiares especialistas en preparación de cadáveres y de las ceremonias que deben ser llevados a cabo. Lo bañan y preparan para que se conserve por la cantidad de días necesarios, en espera de los familiares que se encuentran fuera del lugar del fallecido. Para estos casos utilizan seville orange - naranja agria, nuez moscada, cal, y otras especies y en una tela blanca, colocan a la persona, le ponen un pañal antes de vestirlo y sobre el estómago depositan granos de café o un platillo con sal.

Se acostumbra vestir a los menores de edad con ropa blanca y a los adultos con un traje que ha sido elegido por un familiar cercano para la ocasión, o en su defecto, ropa nueva, si así lo deciden. Al varón, se le viste generalmente con camisa blanca manga larga, pantalón negro, pañuelo blanco, corbata y calcetines. A las mujeres, con un vestido de su preferencia previamente seleccionada por ella, si el tiempo se lo permitió, o con un vestido nuevo, de colores bajos, usualmente blanco, celeste, amarillo o rosado pálido, y en algunos casos se cubre el rostro con un velo blanco de encaje.

Hoy día, muchas personas optan por utilizar formalina para la preparación del cuerpo. Usualmente se auxilian de una enfermera de su confianza para que los apoye 
en la preparación de la persona fallecida. En caso de rigidez del cuerpo durante el proceso de baño y vestimenta, se procede a hablar con la persona fallecida pidiendo su ayuda para que el cuerpo se pueda poner suave, liviano y de fácil manejo. Antes de colocarlo en el ataúd y trasladar el cuerpo a la sala, familiares o amigos que padecen de alguna enfermedad respiratoria, se acercan a la cama o al ataúd abierto y de manera silenciosa le solicitan que se lleve con ellos su enfermedad.

Una vez trasladado el cuerpo a la morada final, se procede a cambiar las sábanas que estaban puestas por unas blancas, las cuales son removidas hasta el ritual de los nueve días. Los familiares, vecinos y amigos cercanos aseguran que la casa habitacional y el predio estén limpios y las cosas de valor guardadas. Proceden a acondicionar el lugar para la vela, ubicando sillas y mesas en distintos lugares del patio y de la casa para recibir a los acompañantes. Arreglan la casa con cortinas blancas, manteles blancos, cubriendo los espejos, ventanas o cualquier otra superficie reflectora para que el alma del fallecido no quede atrapada en la casa.

La responsabilidad de dar aviso a la Iglesia recae en un familiar o vecino cercano. El campanero, inmediatamente, repica con un toque de difunto para avisar el fallecimiento de un miembro de la comunidad, a la población, a través de un sonido de campanas muy lento, ceremonioso y espaciado. Cuando fallece un líder religioso, sea este activo o jubilado, el toque de campanas es por un tiempo más prolongado.

Al escuchar el toque de campanas, los vecinos y la comunidad en general, se acerca a la Iglesia o a los vecinos para preguntar sobre la identidad de la persona fallecida, lo cual es prontamente difundida de boca en boca, caminando la palabra. Muchos ancianos acostumbran esperar en el corredor de su vivienda, para recibir información al respecto. La pregunta ¿Quién ha muerto? recorre el pueblo y se comunica la noticia de manera oral o a través de los medios radiales y redes sociales teniendo mucho cuidado al notificar a los familiares que viven fuera de la localidad.

A partir de la notificación algunos miembros de la comunidad, se presentan para hacer la visita. Pero, es hasta en la noche donde gran parte de las personas asisten al velorio. No obstante, desde el fallecimiento de la persona, la casa presenta signos evidentes de que en su interior hay una persona fallecida. Los entrevistados señalan que todavía se considera imperdonable la ausencia no justificada de la familia más cercana, por lo que se movilizan todos los recursos necesarios para que tenga conocimiento y asistan al entierro.

Los entrevistados señalan que, en décadas anteriores, a la persona fallecida se le mantenía en la cama durante la vela en tanto los amigos y vecinos se encargaban de hacer el ataúd, la cual debía estar terminada antes del amanecer. Era un trabajo colectivo, algunos se encargaban de buscar los materiales para hacer el empaque del 
ataúd, la almohada, barniz, entre otras cosas. Otro grupo se encargaba de cavar el hoyo o hacer la tumba de concreto en el cementerio.

En la construcción del ataúd, se usaban maderas finas como caoba, cedro real o laurel y el vecino es el que generalmente aseguraba su construcción. Las personas encargadas de esta tarea solicitaban en el aserradero la cantidad de madera que necesitaban y les era entregada sin costo alguno. Asimismo, si la casa de los familiares del fallecido es de madera y se encuentra en mal estado, se gestionaba la madera para reparar la baranda y el piso, tomando en cuenta que tendrían la visita de muchas personas y que debía de estar en buenas condiciones para resistir el peso de la gente y evitar posibles accidentes.

Otro grupo de personas se encargan de gestionar o comprar suficiente tela para el forro del ataúd, que es generalmente seda lisa o corrugado. Una vez concluido el ataúd, se le entrega a la persona designada para trabajar en el acabado interior del mismo. En tanto, familiares les garantiza alimentos y bebidas a los que cavan el hoyo o construyen la bóveda. En estos momentos difíciles, lo primordial es el acompañamiento al difunto y sus familiares, compartiendo comida y conviviendo en el hogar. $\mathrm{Al}$ velorio asisten vecinos y amigos quienes llevan consigo comida, bebida o dinero en efectivo para aportar a los gastos fúnebres.

El difunto es despedido por sus familiares, amigos, conocidos, compañeros de trabajo, entre otros, quienes además se acercan a la familia allí presente para brindarles su pésame y organizarse para las diferentes tareas a realizar durante el set up (velorio). Los vecinos y amigos se organizan, conforme la especialidad de cada quien como una red de apoyo. En caso que los adultos por motivos de enfermedad no pudieran hacer acto de presencia a la casa del fallecido, envían a sus hijos o nietos para apoyar en los quehaceres y así los familiares puedan llevar el luto con mayor tranquilidad y sin mayor preocupación. Práctica que aún se mantiene en el Pueblo Afrodescendiente en la ciudad de Bilwi, Puerto Cabezas.

Si la persona fallecida es miembro comulgante de la Iglesia Protestante (morava, anglicana, bautista o adventista) el líder religioso acompaña a los familiares por un periodo de dos a tres horas mediante lectura de pasajes bíblicos e himnos religiosos llamados sankey. Si el fallecido no era miembro de ninguna Iglesia, la oración y el set up lo dirige algún familiar que tiene conocimiento sobre el ritual, se encarga de dirigir el canto, toma asiento frente al ataúd y en una mesita coloca su bebida y un recipiente con sal. A viva voz da la primera frase de la estrofa del himno para que los presentes le den continuidad a través del canto. Para ello es importante que la persona conozca la letra ya que no utiliza himnario, ni folleto (song sheet) para tal efecto.

Esta ceremonia se realiza con evocación de dolor y pena en donde los cantos de liberación de los esclavos se transforman en cantos de liberación del alma. También se 
acostumbra escuchar música religiosa tradicional como Pass me not, o gentle Saviour, the old rugged cross, amazing grace, what a friend we have in Jesus por mencionar algunos. En Puerto Cabezas, Mr. Milton “Taiga” Omier, le gustaba cantar al son de su guitarra.

Tradicionalmente se sirve journey cake, pan de coco con mantequilla y queso amarillo (de acuerdo a las posibilidades), sin faltar los light cakes y pot cakes (de yuca, quequisque, arroz, torta de leche, entre otros) acompañado de bebidas calientes como el chocolate, te de jengibre o café, los que se sirven con intervalos de tres horas. Para los que acompañan a los deudos hasta el amanecer, se les garantiza otro servicio de una bebida y comida caliente a las cinco de la mañana.

$\mathrm{Al}$ retirarse del velorio, las personas se van sin despedirse (sin dar las buenas noches o buenos días) ya que existe la creencia que el espíritu del fallecido o duppy lo puede seguir hasta su casa, siendo éste, un mal augurio para él y su familia.

En los velorios creoles, se asegura que los acompañantes tengan a su disposición juegos de mesa como el dominó, naipe, con la finalidad de mantenerlos despiertos, acondicionando para tal efecto el predio de la casa. Como excepción, ya entrada la noche a los asistentes que acompañan a la familia hasta el amanecer, se les ofrece trago de licor "shots" y cigarros con mucha cautela, garantizando que no se embriaguen.

El velorio, por lo tanto, es un ritual de solidaridad, comunitariedad y de acompañamiento, al difunto y sus deudos durante un lapso de tiempo determinado hasta que sea llevado a su morada o descanso final. Esta obligación de la comunidad con la familia expresa un fuerte sentido de reciprocidad entre los miembros del grupo social. Los desacuerdos, conflictos, enemistades o cualquier otra actitud contraria al de mantenimiento y orden en la comunidad, quedan en suspensión mientras se realizan estos rituales.

\section{El vaso con agua}

En el cuarto del fallecido se coloca un vaso con agua que permanece nueve noches sin cambiarla, por si el alma del difunto tiene sed podrá ir allí a tomarla. Si el agua mantiene su nivel o lo pierde apenas, significa que su alma está descansando y que no necesita de otro ritual. Si el agua va bajando de forma acelerada significa que el alma no está descansando y que ronda por la casa por lo que se necesita buscar ayuda del líder religioso y/o espiritual para realizar otro ritual.

Algunas personas entrevistadas refieren que el agua que queda en el vaso, tiene una connotación medicinal y se ofrece a las personas que sufren de asma, del corazón y de los nervios. La ciencia convencional habla de una evaporación durante este tiempo, mientras que el Pueblo Afrodescendiente tiene la firme creencia que la disminución del agua se debe a que el alma del difunto se hizo presente para beberla. 


\section{Ritual del entierro}

El entierro es la ceremonia espiritual o religiosa donde se procede a dar sepultura al ser querido. En este proceso, se interrelacionan muchos actores: la familia, la comunidad y la Iglesia, este último, no siempre determina la forma en que se desarrollará el ritual.

El ritual inicia con un encuentro en la casa de habitación, en donde el líder religioso y los presentes cantan algunos himnos por un período de treinta minutos para luego dirigirse a la Iglesia. La hora del servicio religioso es consensuada entre las partes. Si la persona no es miembro comulgante, después de ese servicio se dirigen al cementerio para darle sepultura.

Antes de la salida del ataúd de la casa, los familiares aprovechan estos últimos momentos para darles el último adiós, ya sea de manera individual o colectiva, le dicen que es tiempo de partir y a continuación, proceden a colocar en el ataúd objetos muy apreciados por el fallecido para ser utilizado en su viaje a otra esfera. Algunos ponen moneda en el ataúd, para el pago a la entrada al lugar donde habitan los muertos cuando no comulgaban con una Iglesia.

En las familias Afrodescendientes a los menores no se les permiten ver al fallecido. Sin embargo, antes de la salida del ataúd de la casa de habitación se acostumbra pasar a los niños y niñas de la familia por encima del mismo, de un lado a otro por tres veces consecutivas para evitar que sean propensos a enfermedades, morir a corta edad y para evitar que el fallecido se lleve el alma de los niños, o mueran pocos meses después de él. Si no los pasan por encima del muerto, este siempre los recuerda y se los trata de llevar.

Los pies del difunto deben de salir primero de la casa en camino al entierro, para que su frente esté en una dirección diferente al de la casa. La creencia consiste en confundir al espíritu del fallecido para que no pueda encontrar con facilidad el camino de regreso a su casa y poder ir a descansar en paz. Familiares y amigos proceden a cargar el ataúd ya sea a mano entre varios o en un vehículo camino al cementerio. Una vez que el cuerpo este afuera de la casa listo para emprender el viaje, se procede a tocar la campana de la Iglesia para que los vecinos sepan que la procesión va en camino a la Iglesia.

Al ser el entierro un evento formal, se debe hacer uso de la vestimenta adecuada que por lo general, es blanco y negro combinado o totalmente negro; y con menos frecuencia los colores morado y blanco. Los hombres utilizan pantalón negro, camisa blanca y corbata y las mujeres vestidos o falda con blusa, algunas veces pantalón. Si una persona asiste al entierro con ropa de colores vivos, se considera una ofensa o irrespeto hacia el fallecido y sus familiares, convirtiéndose en el centro de atención y comentarios negativos. 
Una vez en la Iglesia, se acostumbra que las personas se pongan de pie, en posición firme como señal de respeto al fallecido al entrar y salir el féretro. Este acto de celebración de vida es previamente consensuado entre el Reverendo y el o la jefa de la familia. La reflexión espiritual constituye una parte integral del servicio religioso, en algunos casos se incluye un pasaje favorito del fallecido. Cada servicio es único, la duración de la misma va en dependencia de los factores acordados entre el celebrante y la familia. Uno de los familiares se encarga de leer su biografía, resaltando sus buenas obras y enseñanzas de vida que pueda servir de ejemplo para los presentes. Se aprovecha el momento para agradecer las muestras de cariño y acompañamiento en estos momentos difíciles.

Dependiendo de la religión, se procede a leer la liturgia "The burial of the Dead" (E1 entierro de los muertos) o algún texto similar que contenga en sus partes conducentes el Padre Nuestro, haciendo énfasis en que no vivimos, ni morimos para nosotros mismos, sino que vivimos y morimos en Cristo, quien murió y resucitó como Señor de los vivos y de los muertos. El funeral se caracteriza por la celebración de la vida, en el cual los hermanos en Cristo guardan luto por la pérdida de su ser querido, quien ha entrado en la presencia del Salvador, como un indicativo de su creencia de la existencia de una vida celestial después de la muerte.

En esos momentos, se suspende la lectura de la liturgia y se procede a retirar el ataúd de las instalaciones de la Iglesia para dirigirse al campo santo en el siguiente orden: Al frente del cortejo se encuentra el Reverendo de la Iglesia y los familiares más cercanos del difunto, sea su pareja o uno de sus hijos, luego el coche fúnebre, después los demás familiares, amigos y allegados, que acompañan.

Familiares y amigos descargan el ataúd en el lugar donde se realizará la despedida final, continuando el líder religioso con la lectura de la letanía, elevando una oración al Altísimo. Es una reflexión sobre la vida, la muerte y el afterlife (inmortalidad). Se invita a los presentes para el last viewing (última visualización) antes de depositar sus restos en la bóveda o directo a la tierra en tanto los presentes entonan los himnos. Usualmente los presentes llevan agua, toalla, o agua de florida en caso de que alguien se desmaye durante este último adiós. Para ello, utilizan el agua de florida para disminuir la tensión, relajar los músculos y sobre todo que la persona recobre consciencia.

Es un momento de despedida del cuerpo físico y con el que se encontrarán en otra vida. Se llora en silencio o en voz baja ya que demostraciones de dolor a viva voz son interpretados como cargo de consciencia, remordimientos que se tiene cuando ha procedido mal en algún momento de su vida y que no pudo enmendar dicho error con la persona fallecida. Usualmente el Reverendo eleva una última oración para cerrar el ritual del entierro. Ante este hecho trascendental, todos reaccionan de forma diferente estableciendo mecanismos para sobrellevar el dolor que conlleva la pérdida de un 
ser querido, la misma está íntimamente relacionada con nuestra cultura, creencias y tradiciones que juegan un papel preponderante en la vida de cada uno de nosotros.

\section{"Nine night": La despedida del alma}

Se desarrolla en un primer momento mediante una ceremonia en donde familiares se reúnen para recordar al fallecido a través de cantos, anécdotas y en algunos casos, se eleva una oración al Altísimo por el alma del fallecido.

En el segundo momento, prevalecen las creencias y prácticas ancestrales, mediante el ritual de despedida del alma. Se cree que tarda nueve días en regresar a África, para su descanso eterno, momento en que finalmente hace la transición al afterlife (después de la vida) la cual es jerarquizado por la persona que preparó al fallecido. En este proceso, se procede a limpiar el cuarto y doblar las cortinas, fundas y sábanas que permanecieron puestas por los nueve días posteriores a su fallecimiento y después proceden a voltear el colchón.

La finalidad de este ritual es que el fallecido no reconozca el lugar, instando a su espíritu a partir para ir a descansar. En estos momentos se procede a cantar "Sleep on, beloved, sleep, and take thy rest" (Duerme, querida, duerme y toma tu descanso) y en casos especificos "Sweeping through the gates of the new Jerusalem, Washed in the blood of the Lamb" (Barriendo a través de las puertas de la nueva Jerusalén, lavado en la sangre del Cordero), en caso de que el espíritu sigue presente y se quiere evitar que el duppy o espíritu de la persona fallecida, permanece en este mundo como un alma perdida al no encontrar el camino al más allá.

El noveno día es también una etapa de reflexión en donde se recuerda lo mejor de los fallecidos, se hace referencia a las cosas que hacía y como era en vida. Después del nine-night la familia cercana se reúne y se distribuyen las pertenencias del fallecido como recuerdo. Se le da la oportunidad a cada una de las partes de escoger un bien, el cual tiene un valor más sentimental que económico. El resto de bienes, quedan para la persona que habita la casa, quien decide si se queda con ellas, las distribuye entre los vecinos o las dona a la Iglesia para obras sociales.

\section{El luto}

El luto, es la expresión externa del dolor por la pérdida de un ser querido. Se guarda como señal de respeto, solidaridad y homenaje a la persona fallecida. Se espera que los dolientes se vistan sólo de color negro o blanco y negro por un periodo usualmente de tres a seis meses, aunque algunas personas guardan luto por más de un año dependiendo del grado de afinidad o consanguineidad con la persona fallecida. Refieren las personas que realizan trabajos formales, que el Código Laboral solamente 
les otorgan tres días de permiso debiendo regresar a su rutina diaria. Por tanto, existe una flexibilidad de colores en la vestimenta durante este periodo.

Se acostumbra suspender por el período de luto la participación en actividades recreacionales. Pero, en reuniones familiares se cuentan historias, chistes y anécdotas de los tiempos de la juventud, haciendo referencias de la persona, tratando de recordar la persona y tener presentes a nuestros seres queridos con alegría, y el amor que los caracterizaba.

Cuando alguien muere, los familiares permanecen tristes por un tiempo, hasta que logran recuperarse. Se tiene a los amigos, vecinos, a la comunidad apoyando la organización de la vela, el entierro, los nueve días, llevando horneados, queques, café, y diferentes cosas sin preguntar si lo quieren o lo necesitan, simplemente lo llevan, eso demuestra que están con ellos, a lo que llaman solidaridad. Los entrevistados afirman que cuando se les acompaña con cantos, ayuda a llevar el luto de una mejor manera. Se sabe que el ser querido debe partir, que se hizo lo que se pudo y por lo tanto, mañana se debe acompañar a otra persona en esos momentos difíciles. La lección aprendida es la espiritualidad, solidaridad, acompañamiento y la comunitariedad que se practica por el bienestar de los miembros de la comunidad.

\section{Ritualidad del año}

El Pueblo Afrodescendiente no acostumbraba realizar aniversario de año, todo culminaba con el nine night. Como Creoles, se tiene la práctica de atender y hacer todo lo posible por la familia cuando están vivos y hay que saber desprenderse del ser querido cuando muere y dejarlos partir y descansar en paz, refieren las entrevistadas. La práctica del One Year Aniversary es algo reciente, se realiza en la Iglesia o en la casa habitacional y es opcional.

\section{Conclusiones}

A través de la historia oral, se recuperaron relatos, testimonios, historias de vida y se conocieron detalles de la espiritualidad, valores, cultura y significados que el pueblo Afrodescendiente otorga a este ritual desde la práctica, como un aporte a la recreación de conocimientos, saberes y haceres. Se complementó con algunas fuentes escritas sobre rituales de entierro en Pueblos y Comunidades Afrodescendientes alrededor del mundo. Lo anterior, para constatar la existencia de similitudes entre ellos, y conocer el recorrido desde la visión de estos pueblos tomando en cuenta la interculturalidad, espiritualidad y complementariedad de las prácticas culturales.

En el presente trabajo, se identificaron cinco hitos en el duelo Afrodescendiente que inician con las señales o anticipos, fallecimiento y velorio en casa, entierro, nueve días y culmina en algunos casos con la ritualidad de año. Sobresale el apoyo 
de la red comunitaria en la organización de las ceremonias y rituales desarrollado durante el proceso de agonía, muerte, duelo y entierro de un fallecido, destacando la espiritualidad, comunitariedad, solidaridad y acompañamiento a la familia de manera desinteresada como un proceso de buena práctica necesaria de mantener vivo, como parte de la tradición del Pueblo Afrodescendiente.

\section{Lista de referencias}

Allue Martínez, Marta (1982). Rituales Funerarios. Institut Catala d' Antropología, Tarragona. Axiu D’etnografia de Catalunya.

Moravian Church in America, Northern and Southern Provinces (1969). Hymnal and Liturgies of the Moravian Church (Himnario y Liturgias de la iglesia Morava). Publicado por la Autoridad del Sínodo Provincial de la Iglesia Morava en América.

UNESCO (1982). Conferencia Mundial sobre las políticas culturales, México. 